\title{
A Case of Milky Urine Due to Chyluria
}

NUM Arif ${ }^{1}, \mathrm{R}$ Biswas ${ }^{2}$, W Zaman ${ }^{3}$

\begin{abstract}
:
Chyluria is an uncommon condition characterized by lymphatic fluid excretion into the urinary tract. Most common cause of chyluria is the infection by parasites e.g. filariasis. Medical treatment is often insufficient to treat the cases of chronic chyluria for which the standard treatment is surgical renal pedicle lymphatic disconnection. However, sclerotherapy by instillation of a sclerosant agent into the renal pelvis may represent an alternative to surgery. This technique has the advantage of being less invasive. We are presenting two cases, presented to us with the complaint of milky urine for more than 3 years. All tests for infectious diseases remained negative. Chyluria was confirmed by urinary chyle test and urinary triglyceride. Cystoscopy showed a clear diagnosis of a milky urine jet from ureteric orifice. Retrograde pyelography revealed pyelolymphatic fistula in one case. Patients were treated successfully with renal pelvic instillation of diluted povidone iodine $(0.2 \%)$. solution and non-ionic contrast(omnipac). We used sclerotherapy with povidone iodine solution as a safe, effective and minimally invasive treatment in our patient with chyluria.
\end{abstract}

Keywords: Lymphatic filariasis, Chyluria; Sclerotherapy;

\section{Introduction:}

Chyluria is the passage of chyle in the urine giving a typical milky appearance. Filariasis is the commonest cause of chyluria. Chyluria represents the chronic stage of filarial disease and is seen in $1-2 \%$ of patients of filariasis after 10-20 years of initial infection. Ninety percent of these infections are caused by $W$. bancrofti, and most of the remainder by Brugia malayi. ${ }^{1}$ The lymphatics of the kidney follow the renal vein and end in the lateral aortic

1. Dr. Nazim Uddin Mohammad Arif, Specialist, Department of Urology, Andrology \& Renal Transplatation, Apollo Hospital Dhaka.

2. Dr. Ranen Biswas, Registrar, Department of Urology, Andrology \& Renal Transplatation, Apollo Hospital Dhaka.

3. Dr. Waheed Zaman, Senior Consultant \& Coordinator, Department of Urology, Andrology \& Renal Transplatation, Apollo Hospital Dhaka, Bangladesh,

\section{Corresponding Author:}

Dr. Nazim Uddin Mohammad Arif, Specialist, Department of Urology, Andrology \& Renal Transplatation, Apollo Hospital Dhaka, Bangladesh, arif_bd_ctg06@yahoo.com glands. The efferents from the lateral aortic gland form the lumber trunks. The lumber and intestinal trunks drain into the cisterna chili. Dilatation and varicosity of retroperitoneal, intestinal and renal lymphatic may develop by lymphatic obstruction. Toxins released from dying filarial worms may leads to impairment of valvular mechanism of the lymphatic vessels. Subsequently, chyluria may develop by rupture of these dilated varicous lymphatics into renal calyces and renal pelvis. We are presenting two cases of chyluria, were treated effectively with povidone iodine $(0.2 \%)$ sclerotherapy.

\section{Case report:}

Two patients were presented to our department with the complaint of passage of milky urine. Both of them were male, case-I was 50 years old and case case-II was 63 years old, with history of passage of milky urine for more than 3 years and for more than 15 years respectively. Their symptom used to increase after taking fatty diet. Both of them became mild weaker than previous, otherwise they were clinically stable. Investigation of case -I showed normal eosinophile count, mild proteinuria, urinary chyle test was positive, haematological test for filariasis was negative, x-ray intravenous urography showed bilateral normal excreting kidneys. Investigation of case-II showed normal eosinophile count, urinary chyle test was positive, urinary triglyceride was more than $1833 \mathrm{mg} / \mathrm{dl}$, haematological test for filariasis was negative, x-ray intravenous urography showed bilateral normal excreting kidneys. Cystoscopy of both patients revealed milky urine in bladder \& unilateral milky ureteric jet(case-I right side $\&$ case-II left side)[Fig: 1,2].

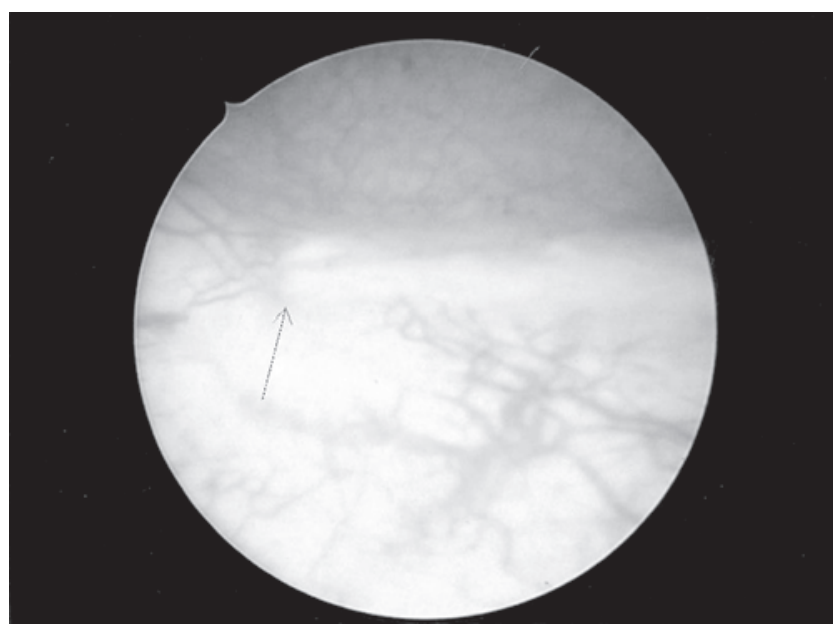

Fig:1: Cystoscopy showing milky urinary jet from left ureteric orifice. 


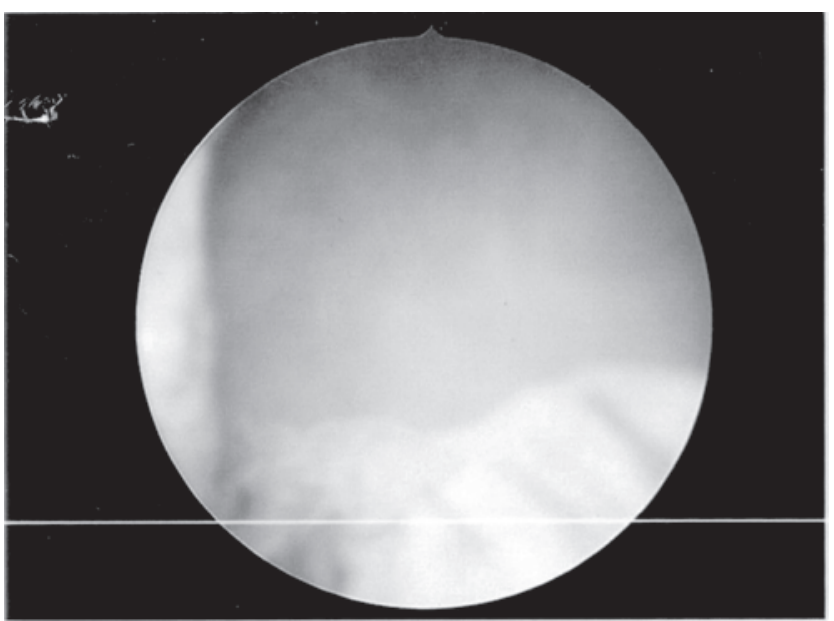

Fig: 2: Cystoscopy showing urinary bladder full with milky urine.

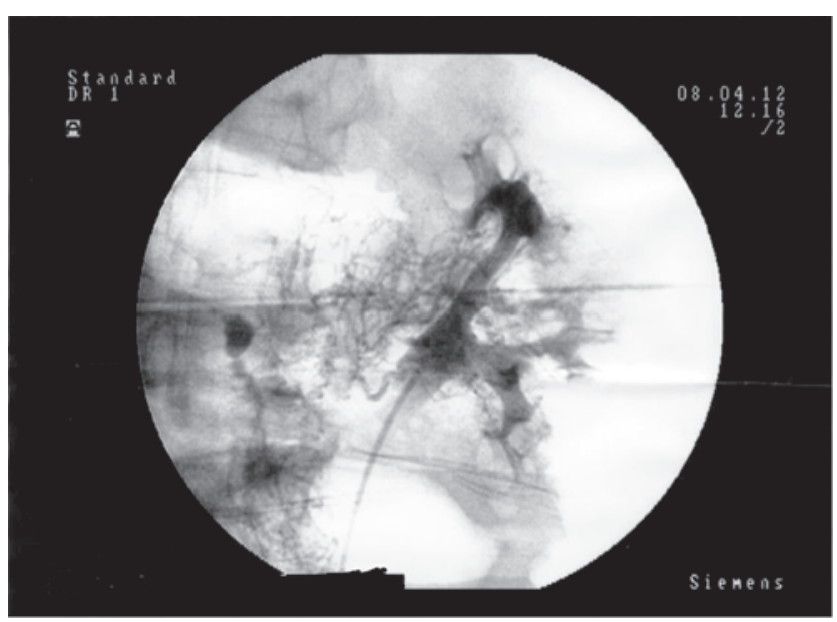

Fig:3: Fig: 3: Retrograde pyelogram showing pyelolymphatic communication.

In case-I retrograde pyelography failed to demonstrate pyelolymphatic fistula. According to right ureteric jet he was diagnosed as a case of chyluria from right kidney. But, in case-two retrograde pyelography showed left sided pyelolymphatic communication[Fig: 3]. Both patient received multiple courses of antifilarial drugs like diethylcarbamizine (DEC) and albendazole previously. Retrograde uretric catheterization \& sclerotherapy with povidone iodine $(0.2 \%)$ was instelled 8 hourly for 3 cosecutive days(total 9 doses). Initially, During retrograde pyelography both the patients received non-ionic contrast also. After sclerotherapy, both patients were on low fatty diet for four weeks. Gradually, their chyluria decreased \& stopped. After 6 months followup both patients were passing clear urine.

\section{Discussion:}

Chyluria and genital oedema is not common and often encountered by the urologists. Chyluria is the passage of chyle into the urine giving it a typical milky appearance, it's due to communication between lymphatic and urinary system. Filariasis is the most frequent cause of chyluria. Chyluria mostly occurs unilaterally and more common on left side. $^{2}$ In chyluria, investigations are aimed to detection and confirmation of the presence of chyle in urine and localization of the lympho-urinary fistula.

Chylous urine is best studied immediately after it has been voided. Fatty diet a day or night before has been used to enhance chyluria. Chylous urine, when kept in the test tube, it usually settled into three layers, the fat being lighter gets deposited as the top layer, the fibrin clots form the middle layer and cells together with debris settle in the bottom layer.

Urinary triglyceride have been demonstrated to be universally present even if the urine is clinically clear. ${ }^{3}$

Prolonged chyluria results loss of weight, hypoprotenemia, lymphopenia and anaemia. Initially medical treatment should be tried in every case, which consists of dietary modification, antifilarial drugs, bed rest and high amount of fluid intake., 4 Chyluria is a debilitating but not life threatening condition. Hence, any treatment of chyluria must be minimally invasive, safe, and effective. Renal pelvic instillation sclerotherapy (RPIS) is a minimally invasive treatment of chyluria. It involves placement of ureteric catheter into the pelvis of the offending renal unit and the renal pelvic capacity is measured after contrast instillation. Silver nitrate and povidone iodine are the most commonly used sclerosants in RPIS. Silver nitrate $(0.1-1 \%)$ is effective in $59.4 \%-83.6 \%$ of cases and povidone iodine $(0.2 \%)$ has shown more efficacy than silver nitrate( $81 \%-100 \%) .{ }^{6}$

Problem with the conservative treatment \& sclerothrapy is disease recurrence. Surgery assumes importance in such refractory cases. Study showed that $20 \%$ of chylura patient required surgery. ${ }^{7}$ The indications are a weight loss, hypoproteinemia, anasarca, recurrent clot retention, hematochyluria, recurrent urinary tract infection because of

\section{Conclusion:}

Chyluria is a rare and quite debilitating disease due to loss of fat in the urine. The sclerotherapy has been found to be safe, effective and minimally invasive treatment for chyluria. ${ }^{6}$ We used povidone iodine as effective solution for sclerotherapy in our patient with chyluria.

1. Diamond Eric, Schapira ME, Chyluria. A review of literature, Urology 1985;26:427-31.

2. Koo CG, Langenberg V. Chyluria: A clinical study. J Roy Coll Surg 1971;14:3.

3. Johnston DW. Chyluria. Case report \& review of literature. Ann Int Med 1955;42:931.

4. Hashin S, Rohol HB, Babayan VK, Vanitallie TB. Treatment of chyluria and chylothorax with medium chain triglyceride. N Eng J Med 1964;270:756-61.

5. Geliebter A, Torby N, Bracco EF. Over feeding with medium-chain triglycerides diet results in diminished deposition of fat. Am J Clin Nut 1983;37:1-4.

6. Kj Singh, A Srivastava. Nonsurgical management of chyluria (sclerotherapy). Indian J. Urol 2005; 21(1) : 55-58.

7. Karanjavala DK. Technique of clearance (or disconnection) of dilated lymphatics in the renal hilum and lower ureter and bladder in cases of intractable chyluria or haemochyluria. Br J Urol 1979; 51:440-2. 